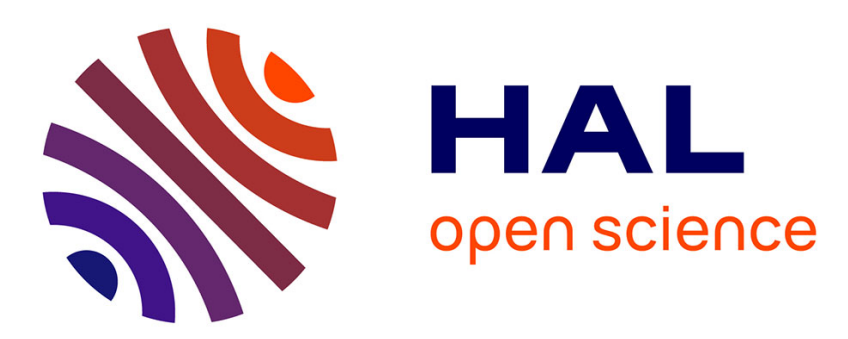

\title{
Beyond universality: Parametrizing ultracold complex-mediated reactions using statistical assumptions
}

Manuel Lara, P. G. Jambrina, Jean-Michel Launay, F. J. Aoiz

\section{To cite this version:}

Manuel Lara, P. G. Jambrina, Jean-Michel Launay, F. J. Aoiz. Beyond universality: Parametrizing ultracold complex-mediated reactions using statistical assumptions. Physical Review A : Atomic, molecular, and optical physics [1990-2015], 2015, 91 (3), pp.030701-1.5 (R). 10.1103/PhysRevA.91.030701 . hal-01135517

\section{HAL Id: hal-01135517 https://hal.science/hal-01135517}

Submitted on 25 Mar 2015

HAL is a multi-disciplinary open access archive for the deposit and dissemination of scientific research documents, whether they are published or not. The documents may come from teaching and research institutions in France or abroad, or from public or private research centers.
L'archive ouverte pluridisciplinaire HAL, est destinée au dépôt et à la diffusion de documents scientifiques de niveau recherche, publiés ou non, émanant des établissements d'enseignement et de recherche français ou étrangers, des laboratoires publics ou privés. 


\title{
Beyond universality: Parametrizing ultracold complex-mediated reactions using statistical assumptions
}

\author{
Manuel Lara* \\ Departamento de Química Física Aplicada, Facultad de Ciencias, Universidad Autónoma de Madrid, 28049 Madrid, Spain \\ P. G. Jambrina \\ Departamento de Química Física, Facultad de Química, Universidad Complutense, 28040 Madrid, Spain \\ J.-M. Launay \\ Institut de Physique de Rennes, UMR CNRS 6251, Université de Rennes I, F-35042 Rennes, France \\ F. J. Aoiz ${ }^{\dagger}$ \\ Departamento de Química Física, Facultad de Química, Universidad Complutense, 28040 Madrid, Spain \\ (Received 10 November 2014; revised manuscript received 2 February 2015; published xxxxxx)
}

We have calculated accurate quantum reactive and elastic cross sections for the prototypical barrierless reaction $\mathrm{D}^{+}+\mathrm{H}_{2}(v=0, j=0)$ using a modified hyperspherical scattering method. The considered kinetic energy ranges from the ultracold to the Langevin regimes. A reaction rate coefficient practically constant in no less than eight orders of magnitude is obtained. The availability of accurate results for this system allows one to test the quantum theory by Jachymski et al. [K. Jachymski, M. Krych, P. S. Julienne, and Z. Idziaszek, Phys. Rev. Lett. 110, 213202 (2013)] in a nonuniversal case. The short-range reaction probability is rationalized using statistical model assumptions and related to a statistical factor. This provides a means to estimate one of the parameters that characterizes ultracold processes from first principles.

PACS number(s): 34.10.+x, 34.50.Lf

The increasing availability of cold and ultracold samples 3 of atoms and molecules has sprung great interest in chemical reactions at very low temperatures [1-5]. Although new experimental approaches [5] appear highly promising, advances in the field are hampered by technical problems in producing most molecules at low temperatures and high enough densities. In contrast to neutral species, ions can be easily trapped and cooled. The technology of Coulomb crystals in radiofrequency ion traps [6] and the possibility of combining them with traps for neutrals or with slow molecular beams $[7,8]$ promise great progress in the analysis of ion-neutral reactions in the near future.

Theoretical simulations employing standard $a b$ initio approaches are not feasible for most of the systems thus far considered. For heavy systems (more convenient experimentally) there are no potential energy surfaces (PESs) accurate enough to describe processes near thresholds. Additionally, most of exact dynamical treatments face insurmountable problems in such regimes. However, in contrast to short-range (SR) chemical interactions, those occurring at long range (LR) can be more easily calculated. Moreover, theoretical approaches based only on the knowledge of the LR part of the PES have been able to describe recent experimental findings nearly quantitatively [1,9]. Indeed, processes at very 46 low collision energies favor LR interactions, leading to the 47 idea of universality in extreme cases [10]: the result of the collision depends exclusively on the LR behavior and not on the details of the PES. In this regard, recently proposed 50 LR parametrization procedures [9,11-13] are very appealing.

\footnotetext{
*manuel.lara@uam.es

†aoiz@quim.ucm.es
}

Fitting experimental data, these models are able to predict 51 nonmeasured values providing some insight into the under- 52 lying interactions. In particular, the approach by Jachymski 53 et al., based on multichannel quantum-defect theory (MQDT), 54 provides analytical expressions which can be easily compared 55 with experimental data $[9,14,15]$. The model has been recently 56 applied to a variety of systems $[9,16,17]$. In particular, for the 57 Penning ionization of $\mathrm{Ar}$ by $\mathrm{He}\left({ }^{3} \mathrm{~S}\right)$ [5], the rate coefficients 58 have been fitted in a wide range of collision energies using only $\quad 59$ two parameters [9]. However, the parameters of the model are 60 phenomenological and they had not been determined before 61 from first principles.

In this work, we present accurate calculations for the reac- 63 tive collision $\mathrm{D}^{+}+\mathrm{H}_{2}(v=0, j=0)$ using the hyperspherical 64 reactive scattering method [18]. We consider collision energies 65 that range from the ultracold regime, where only one partial 66 wave is open, to the Langevin regime where many of them 67 contribute. These calculations allow us to test the model 68 by Jachymsky et al. [9,14] by comparison with accurate 69 theoretical results in a realistic atom + diatom system, 70 providing a way to estimate one of the parameters using simple 71 statistical model assumptions, which do not require performing 72 any quantum reactive scattering calculation.

The $\mathrm{H}^{+}+\mathrm{H}_{2}$ system is the prototype of ion-molecule 74 reactions, which are usually nearly barrierless and exhibit large 75 cross sections due to their LR, $\propto-C_{n} / R^{n}, n=4$, potentials. 76 At energies below $\approx 1.7 \mathrm{eV}$, the proton exchange is the only 77 reactive channel, and the process can be described on the 78 ground adiabatic PES [19-22]. Since the PES is characterized 79 by a deep well or complex $(\approx 4.5 \mathrm{eV})$, as illustrated in 80 Fig. 1, rigorous statistical models $[23,24]$ have been applied 81 to this reaction and isotopic variants in the low and thermal 82 energy regimes [20,24-27] in good agreement with accurate 83 


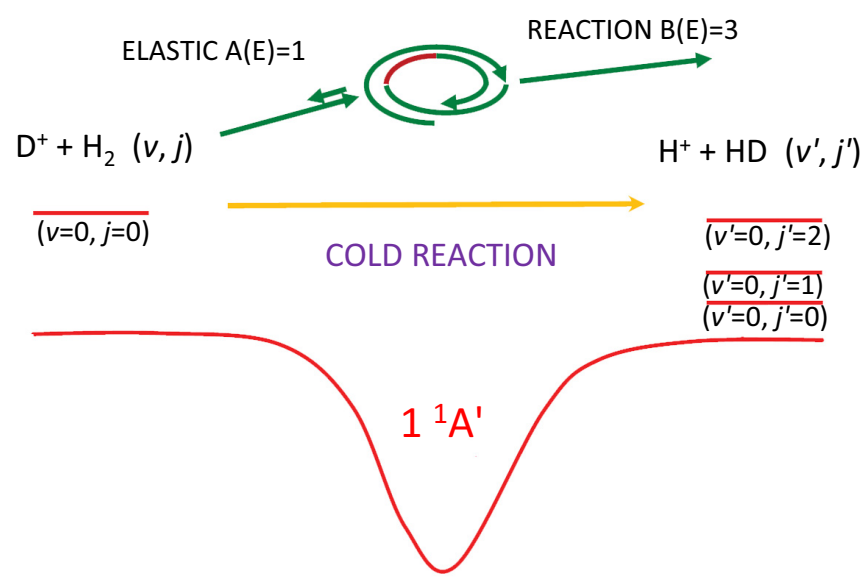

FIG. 1. (Color online) Sketch of the intrinsic reaction path and the rovibrational states involved in the reaction at the studied energies. $A(E)$ and $B(E)$ are the number of incoming and outgoing channels, respectively ( 1 and 3 for $J=0$ ).

34 calculations. Specifically, the $\mathrm{D}^{+}+\mathrm{H}_{2} \rightarrow \mathrm{H}^{+}+\mathrm{HD}$ reaction 85 features a small exoergicity (difference of zero-point energies). Experiments to determine state-specific rate coefficients at energies as low as $12 \mathrm{~K}$ [28] have been carried out, and lower temperatures are expected to be feasible soon [29].

The deep ultracold regime, governed by Wigner laws [30], is described in terms of the scattering length. The latter largely varies with slight changes of the interaction potential. Due to inaccuracies in the state-of-the-art electronic calculations, only for very particular atom + atom systems [31-33] has it been possible to reproduce the experimental scattering length theoretically. As atom + diatom systems are even more complicated, it is difficult to assess the accuracy of calculations for ultracold energies. In the spirit of the work by Gribakin et al. [34], we can consider our study as an effort to determine a "characteristic" scattering length. Besides, assuming that the interaction of the system is reasonably described by the current PES, we can use our results to test recent methodologies, like the approach in Refs. $[9,14]$.

In the (ultra)cold regime both accurate descriptions of the LR interactions and dynamical propagations up to very large distances are two strict requirements. The PES by Velilla et al. [35], which includes the LR interactions in the functional form, satisfies the first requirement. The dominant contributions involve the charge quadrupole, $\propto-R^{-3}$, and the charge-induced dipole, $\propto-R^{-4}$, interactions. However, only the latter contributes to collisions in $j=0 .{ }^{1}$ The second requirement is fully satisfied by the hyperspherical quantum reactive scattering method developed by Launay et al. $[18,36]$, recently modified to allow the inclusion of LR interactions $[37,38]$. These modifications are used here for the first time allowing the propagations up to $10^{5}-10^{6}$ a.u. which are required for a $n=4$ potential.

The reaction cross section, $\sigma_{\mathrm{r}}(E)$, in the $10^{-7}-150 \mathrm{~K}$ energy range is plotted in Fig. 2 and compared with the Langevin

\footnotetext{
${ }^{1}$ The integral $\left\langle j=0\left|P_{2}\right| j=0\right\rangle$ is null, and the contributions from $\sim R^{-3}$ and anisotropic polarization terms vanish [35].
}

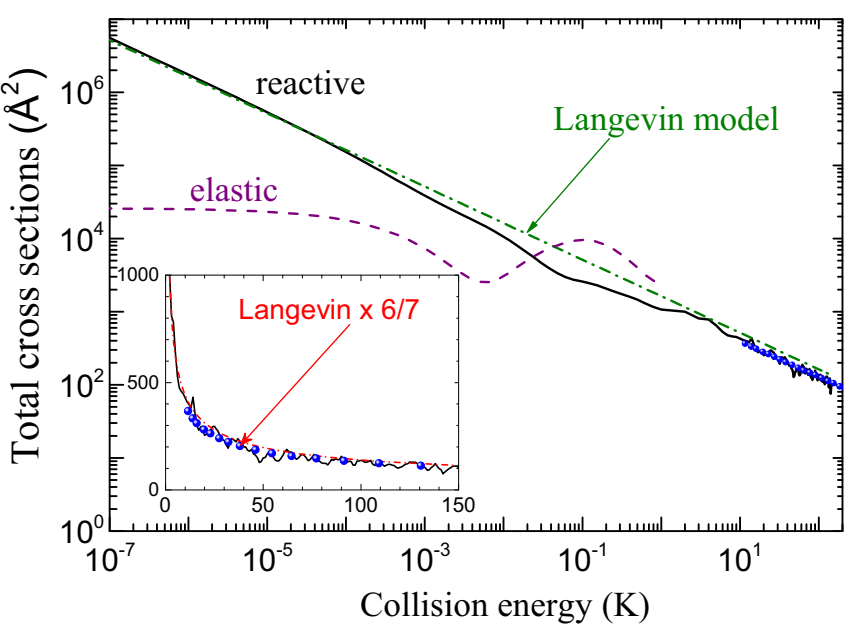

FIG. 2. (Color online) Calculated reaction and elastic total cross section for the collision $\mathrm{D}^{+}+\mathrm{H}_{2}(v=0, j=0)$ compared with the Langevin prediction. The inset shows the comparison of the reaction cross sections with the experimental data [28] and the result of the statistically corrected Langevin model.

model (LM) expression for the cross section, $\sigma_{\mathrm{L}}(E)={ }_{119}$ $2 \pi\left(C_{4} / E\right)^{1 / 2}$. The LM is commonly used to rationalize ${ }_{120}$ collisions in the regime where many partial waves are open. We ${ }_{121}$ have determined the value $C_{4}=2.71$ a.u. using the effective ${ }_{122}$ potential as a function of $R$ which results from averaging ${ }_{123}$ the PES $V(R, r, \theta)$ over $r$ and $\theta$ Jacobi coordinates, with the ${ }_{124}$ $(v=0, j=0)$ probability distribution. The LM implies here ${ }_{125}$ that once the centrifugal barrier is overcome, the system is 126 captured in the complex which subsequently decomposes into ${ }_{127}$ the $\mathrm{H}^{+}+\mathrm{HD}$ arrangement channel with unit probability. The ${ }_{128}$ LM regime can be associated with the high energy part of the ${ }_{129}$ plot (above $1 \mathrm{~K}$, with five partial waves opened). The calculated ${ }_{130}$ cross sections are found smaller than the LM prediction in ${ }_{131}$ this energy range. Indeed, only a fraction of the complexes ${ }_{132}$ decompose into the products.

We can improve the LM using statistical model arguments, 134 which are being revisited in the field of cold collisions [39,40]. ${ }_{135}$ In complex mediated reactions, the statistical ansatz [23,24], ${ }_{136}$ $P_{r}^{J}(E) \approx P_{\text {capt }}^{J}(E) \times P_{\rightarrow \text { prod }}(E)$, can be applied, where $P_{r}^{J}(E){ }_{137}$ is the reaction probability for a given initial rovibrational state ${ }_{138}$ and total angular momentum, $J$ (orbital, $l$, plus rotational, ${ }_{139}$ $j), P_{\text {capt }}^{J}(E)$ is the probability for the reagents to be captured ${ }_{140}$ in the complex, and $P_{\rightarrow \operatorname{prod}}(E)$ is the statistical factor, i.e., ${ }^{141}$ the probability of emerging into the product arrangement 142 channel when the complex decomposes. If there is a complete ${ }_{143}$ randomization of the energy in the complex, the statistical ${ }_{144}$ factor will be independent of the initial state of the reagents, 145 only subject to conservation of energy, $J$, and parity. Roughly ${ }_{146}$ speaking, the fraction of complexes which decompose into 147 the reactants or products is proportional to the respective ${ }_{148}$ number of scattering channels energetically available, denoted ${ }_{149}$ with $A(E)$ and $B(E)$, respectively, considering all of them 150 as equiprobable. Accordingly, the statistical factor can be 151 approximated by $P_{\rightarrow \text { prod }}(E)=B(E) /[A(E)+B(E)]$. At the ${ }_{152}$ considered energies, only three HD rovibrational states are ${ }_{153}$ open, as shown in Fig. 1, and for $J \geqslant 2$ we find that $A(E)=1$ and $B(E)=6$, and $P_{\rightarrow \operatorname{prod}}=6 / 7(\approx 86 \%)$. For $J=0$ and ${ }_{155}$ $J=1$ the statistical factors are $3 / 4(75 \%)$ and $5 / 6(\approx 83 \%), \quad{ }_{156}$ 


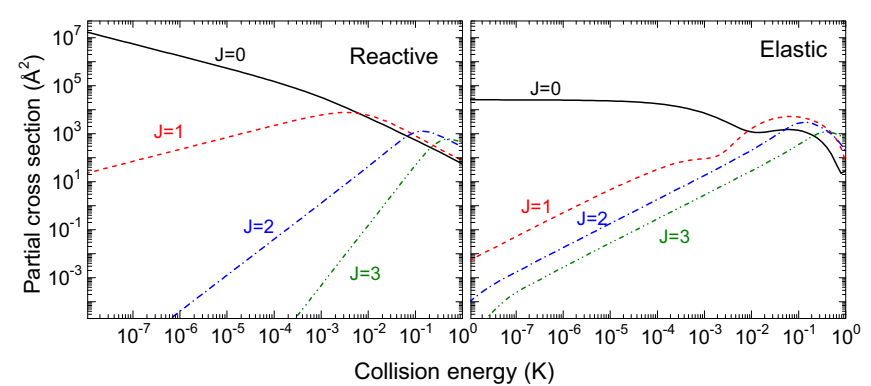

FIG. 3. (Color online) Reaction and elastic partial cross sections at the indicated $J(=l)$ values for the $\mathrm{D}^{+}+\mathrm{H}_{2} \quad(v=0, j=0)$ collision in the cold and ultracold regimes.

157

respectively. If the collision energy is high enough for many partial waves to contribute but low enough for the total number of channels to stay the same, $P_{\rightarrow \operatorname{prod}}(E) \approx 6 / 7$, and

$$
\sigma_{\mathrm{r}}(E) \approx P_{\rightarrow \operatorname{prod}} \sigma_{\mathrm{L}}(E) .
$$

160 Therefore, 6/7 appears naturally as a statistical factor to correct 61 the LM expression. When the number of product channels is 162 large enough, $B \gg A$, then $P_{\rightarrow \text { prod }} \approx 1$ and the result is $\sigma_{\mathrm{L}}(E)$. 63 More accurate statistical implementations, which evaluate $A(E)$ and $B(E)$ as capture probabilities [23,24], lead to similar conclusions.

The inset of Fig. 2 compares the calculated reaction cross section with the experimental data from Ref. [28]. The corrected LM result is also shown and found to be in very good agreement with both the experiment and the present calculations. The similarity of the theoretical results with the experiment is remarkable considering that the latter was performed with $n-\mathrm{H}_{2}$ [21] and lends credence to the predictive power of QM calculations on state-of-the-art PESs for atom + diatom systems ab initio in the Langevin regime.

The cross sections at much lower kinetic energies are also shown in Fig. 2. In the zero-energy limit, Wigner threshold laws [30,41] state that the elastic and the total-loss (inelastic + reaction) cross sections associated with each partial wave, $l$, vary $\sim E^{2 l}$ and $\sim E^{l-1 / 2}$, respectively. However, for a potential with $n=4$, the threshold law for elastic scattering becomes $\sim E$ for any $l>0[30,41,42]$. The ultracold cross sections, shown in Fig. 3 for the four lowest partial waves, comply with these laws (there are no open inelastic channels). The limiting behaviors for $l=0$ are reflected in the total reaction $\left(\sim E^{-1 / 2}\right)$ and total elastic (constant) cross sections in the lowest energy region of Fig. 2, where only the $s$ wave is open.

For $n=4$ the energy dependence of the LM coincides with the Wigner threshold law $\left(\sim E^{-1 / 2}\right)$. Remarkably, the absolute values of accurate and LM cross sections in the ultracold limit are nearly the same, $\sigma_{\mathrm{r}} \approx 1.07 \sigma_{\mathrm{L}}(E)$. Therefore, the reaction rate coefficient (not shown) is practically constant in no less than eight orders of magnitude, and small variations can be further smoothed out with the Boltzmann averaging. In what follows, we will try to rationalize this classical Langevin behavior in the ultracold regime.

Very recently, quantal versions of the LM have been proposed [14,43] under the assumption that all the flux that reaches the SR region leads to reaction. In the $n=4$ case, these universal models conclude that the zero-energy limit of $\sigma_{\mathrm{r}}$ is 200 given by $2 \sigma_{\mathrm{L}}(E)$, and not by $\sigma_{\mathrm{L}}(E)$ as we have approximately 201 obtained. Therefore our system is not universal. The formalism 202 in Ref. [9] is able to deal with systems where the short-range 203 reaction probability, $P^{\text {re }}$, is $<1$. It provides expressions for 204 the complex (energy-dependent) scattering length $\tilde{a}_{l}(k)=205$ $\alpha_{l}(k)-i \beta_{l}(k)$ in terms of the MQDT functions (where $k$ is 206 the relative wave number). This allows us to parametrize 207 $\tilde{a}_{l}(k)$ using two real parameters, $y$ and $s$, together with 208 the mean scattering length [34], $\bar{a}=\left(2 \mu C_{4}\right)^{1 / 2} / \hbar\left(\approx 99.7 \mathrm{a}_{0} \quad 209\right.$ in this case). Specifically, the dimensionless parameter $0 \leqslant 210$ $y \leqslant 1$ characterizes the flux that is lost from the incoming 211 channel at SR, according to $P^{\text {re }}=4 y /(1+y)^{2}$. The Langevin 212 assumption or universal case corresponds to $y=1$. The ${ }_{213}$ dimensionless scattering length $s=\tan (\phi)$ is related to an 214 entrance channel phase $\phi[9,14,42]$.

In terms of these parameters, the small $k$ behavior of the 216 real and imaginary parts of the complex scattering length for ${ }_{217}$ the lowest partial waves $(l=0-3)$ is given by ${ }^{2}$

$$
\begin{gathered}
\alpha_{0}(k) \rightarrow \bar{a} \frac{s\left(1-y^{2}\right)}{1+s^{2} y^{2}}, \quad \beta_{0}(k) \rightarrow \frac{y\left(1+s^{2}\right) \bar{a}}{1+s^{2} y^{2}}, \\
\alpha_{1}(k) \rightarrow-k \bar{a}^{2} \frac{\pi}{15}, \quad \beta_{1}(k) \rightarrow \frac{y\left(1+s^{2}\right) k^{2} \bar{a}^{3}}{9\left(s^{2}+y^{2}\right)}, \\
\alpha_{2}(k) \rightarrow-k \bar{a}^{2} \frac{\pi}{105}, \quad \beta_{2}(k) \rightarrow \frac{y\left(1+s^{2}\right) k^{4} \bar{a}^{5}}{2025\left(1+s^{2} y^{2}\right)}, \\
\alpha_{3}(k) \rightarrow-k \bar{a}^{2} \frac{\pi}{315}, \quad \beta_{3}(k) \rightarrow \frac{y\left(1+s^{2}\right) k^{6} \bar{a}^{7}}{2480625\left(s^{2}+y^{2}\right)} .
\end{gathered}
$$

Our calculations yield the $S$ matrix as a function of the ${ }_{219}$ energy for each total angular momentum $J$ and hence $l$ (for 220 $j=0$ ), which allows us to calculate directly $\tilde{a}_{l}(k)$ using the 221 elastic element of the $S$ matrix [44].

Figure 4 depicts the energy dependence of $\alpha$ and $\beta$ for ${ }_{223}$ $J=0-3$. The limiting behaviors are in perfect agreement with 224 the threshold laws and the power of the dependence on $k$ in 225 Eqs. (2)-(5).

To extract the model parameters $s$ and $y$ from the scattering ${ }_{227}$ results, let us consider first the case $l=0$. Using the values 228 $\alpha_{0}$ and $\beta_{0}$ at the lowest energy given by our calculations ${ }_{229}$ and solving Eqs. (2) for $y$ and $s$, we obtain $y(l=0)=0.35 \quad 230$ and $s(l=0)=-0.82$, which leads to $P^{\text {re }}(l=0)=77 \%$. The ${ }_{231}$ parametrization for higher values of $l$ is not straightforward. 232 The real part, $\alpha_{l}(k)$, is independent on $s(l)$ and $y(l)$, and with ${ }_{233}$ the sole expression of $\beta_{l}(k)$ it is not possible to solve for the ${ }^{234}$ values of the two parameters.

Analogous to the procedure of Ref. [9], assuming that $y{ }_{236}$ and $s$ do not depend on $l$, we can introduce $y(l=0)$ and ${ }_{237}$ $s(l=0)$ in Eqs. (3)-(5) and compare the resulting values of $\beta_{l}{ }_{238}$ with those obtained in the scattering calculations. The ratios of ${ }_{239}$ the calculated and parametrized values of $\beta_{l}$ are $0.4,1.4$, and 240 0.7 for $l=1, l=2$, and $l=3$, respectively. The agreement 241 can be considered good on average, taken into account the 242

\footnotetext{
${ }^{2}$ Equations (2) and (3) were kindly provided by the authors of Ref. [9]; Eqs. (4) and (5) were deduced by the authors of this work following Refs. [9,42].
} 

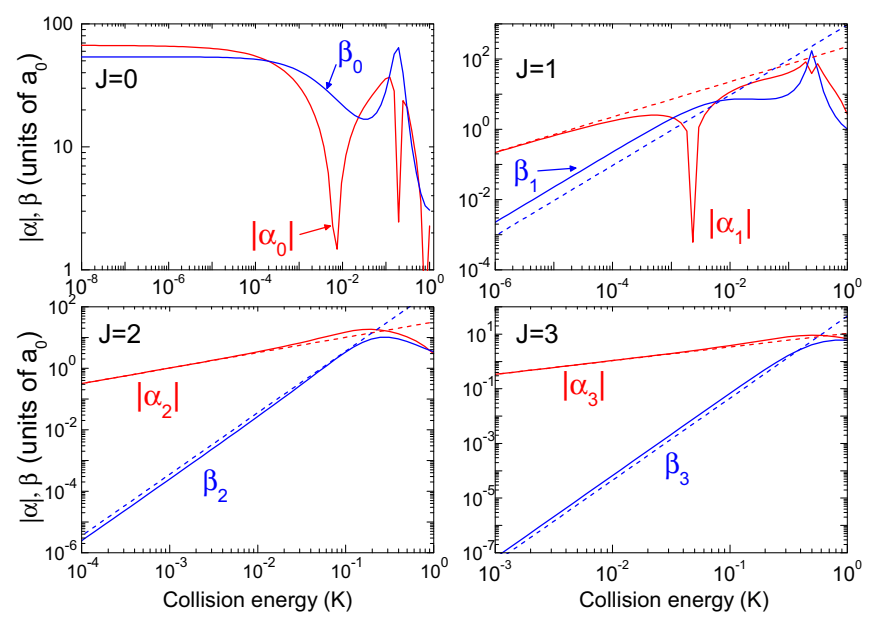

FIG. 4. (Color online) Real and imaginary parts of the scattering length, $\tilde{a}_{l}(k)=\alpha_{l}(k)-i \beta_{l}(k)$, obtained in the calculations for the four lowest partial waves. The absolute value of $\alpha$, mostly negative, is plotted. The values for $J=1,2$, and 3 (in continuous lines) are compared with the predictions from the model in Ref. [9] (in dashed lines), calculated using Eqs. (3)-(5), assuming for $s$ and $y$ the same values that have been obtained for $J=0$.

243 oscillations of this ratio about 1 . As for the real parts $\alpha_{l}$, 244 given by Eqs. (3)-(5), they can be directly compared with our 245 scattering results. The agreement (within 1\%) is very good, 246 which can be deemed as a test of the theory and serves to 247 ensure the convergence of the scattering calculations. These 248 expressions depend only on $\bar{a}$ (not on $s$ or $y$ ) and they can be 249 considered as really universal.

According to Ref. [9], when the dependence of $P^{\text {re }}$ with the 251 energy and $l$ is weak, the expression

$$
\sigma_{\mathrm{r}}(E) \approx P^{\mathrm{re}} \sigma_{\mathrm{L}}(E)
$$

252 is valid in the Langevin regime, $P^{\text {re }}$ being the same value which 253 governs the ultracold behavior. The fact that $\sigma_{\mathrm{r}}(E) / \sigma_{\mathrm{L}}(E)$ has an average value of 0.78 in the range $1-150 \mathrm{~K}$, very close to ${ }_{254}$ the $P^{\text {re }}(l=0)=0.77$ obtained at ultracold energies, indicates 255 a weak dependence of $P^{\text {re }}$ with $l$ and energy and hence the ${ }_{256}$ validity of the assumption of constant parameters made above. 257

Therefore, Eq. (1), obtained from the statistical hypothesis, 258 and Eq. (6), from Ref. [9], are both valid in the Langevin 259 regime. This leads to the equivalence of the fraction of captured 260 flux that reacts, $P^{\text {re }}$, and the fraction of formed complexes 261 which decompose to give the products, $P_{\rightarrow \text { prod }}$, and thus a 262 way to estimate the $P^{\text {re }}$. In fact, $6 / 7(\approx 86 \%)$ is a rough 263 estimate of $P^{\mathrm{re}}=77 \%$, and only requires counting states. A 264 more accurate estimate can be obtained using the ab initio 265 QM values of $\sigma_{\mathrm{r}}(E)$ in the Langevin region to calculate 266 $\sigma_{\mathrm{r}}(E) / \sigma_{\mathrm{L}}(E)$. This second way to estimate $P^{\text {re }}$ does not require ${ }_{267}$ the system to behave statistically and is more general. Finally, 268 the fact that our calculations are expected to be quantitative 269 in the Langevin regime leads to an interesting conclusion: we 270 have found a way to estimate $P^{\text {re }}$, and through it $y$, one of 271 the parameters which characterize the experimental ultracold 272 behavior, while working at much higher energies where 273 state-of-the-art ab initio reaction dynamics is quantitative and 274 require less demanding QM calculations. 275

In summary, accurate scattering calculations have allowed 276 us to test the quantum theory by Jachymsky et al. $[9,14,15] .277$ On average, the same $P^{\text {re }}$ describes the ultracold and the 278 Langevin regimes. We have found a link between $P^{\mathrm{re}}$ and the ${ }_{279}$ statistical factor of the statistical approach to reactions [23]. 280 Apart from physical insight, our analysis provides ways to 281 estimate the parameter $y$, which characterizes the experimental 282 ultracold behavior, using ab initio reaction dynamics at much 283 higher energies, where such methodology is assumed to be 284 quantitative.

The authors are greatly indebted to K. Jachymski, A. 286 Simoni, and T. González-Lezana for fruitful discussions. The ${ }_{287} \mathbf{F Q}$ Spanish Ministry of Economy and Competitiveness (Grants 288 CSD2009-00038, and CTQ2012-37404-C02) are gratefully 289 acknowledged.
[1] S. Ospelkaus, K.-K. Ni, D. Wang, M. H. G. de Miranda, B. Neyenhuis, G. Quéméner, P. S. Julienne, J. L. Bohn, D. S. Jin, and J. Ye, Science 327, 853 (2010).

[2] P. Staanum, S. D. Kraft, J. Lange, R. Wester, and M. Weidemüller, Phys. Rev. Lett. 96, 023201 (2006).

[3] N. Zahzam, T. Vogt, M. Mudrich, D. Comparat, and P. Pillet, Phys. Rev. Lett. 96, 023202 (2006).

[4] E. R. Hudson, N. B. Gilfoy, S. Kotochigova, J. M. Sage, and D. DeMille, Phys. Rev. Lett. 100, 203201 (2008).

[5] A. B. Henson, S. Gersten, Y. Shagam, J. Narevicius, and E. Narevicius, Science 338, 234 (2012).

[6] S. Willitsch, M. T. Bell, A. D. Gingell, and T. P. Softley, Phys. Chem. Chem. Phys. 10, 7200 (2008).

[7] F. H. J. Hall and S. Willitsch, Phys. Rev. Lett. 109, 233202 (2012).

[8] S. Willitsch, M. T. Bell, A. D. Gingell, S. R. Procter, and T. P. Softley, Phys. Rev. Lett. 100, 043203 (2008).
[9] K. Jachymski, M. Krych, P. S. Julienne, and Z. Idziaszek, Phys. Rev. Lett. 110, 213202 (2013).

[10] P. Julienne, Faraday Discuss. 142, 361 (2009).

[11] S. Kotochigova, New J. Phys. 12, 073041 (2010).

[12] B. Gao, Phys. Rev. A 78, 012702 (2008).

[13] G. Quéméner and J. L. Bohn, Phys. Rev. A 81, 022702 (2010).

[14] Z. Idziaszek and P. S. Julienne, Phys. Rev. Lett. 104, 113202 (2010).

[15] K. Jachymski, M. Krych, P. S. Julienne, and Z. Idziaszek, Phys. Rev. A 90, 042705 (2014).

[16] J. Jankunas, B. Bertsche, K. Jachymski, M. Hapka, and A. Osterwalder, J. Chem. Phys. 140, 244302 (2014).

[17] E. Lavert-Ofir, Y. Shagan, A. B. Henson, S. Gersten, J. Klos, P. Zuchowski, J. Narevivius, and E. Narevicius, Nat. Chem. 6, 332 (2014).

[18] J. M. Launay and M. L. Dourneuf, Chem. Phys. Lett. 169, 473 (1990). 
[19] E. Carmona-Novillo, T. González-Lezana, O. Roncero, P. Honvault, J.-M. Launay, N. Bulut, F. J. Aoiz, L. Bañares, A. Trottier, and E. Wrede, J. Chem. Phys. 128, 014304 (2008).

[20] P. G. Jambrina, J. M. Alvariño, F. J. Aoiz, V. J. Herrero, and V. Sáez Rábanos, Phys. Chem. Chem. Phys. 12, 12591 (2010).

[21] P. Honvault and Y. Scribano, J. Phys. Chem. A 117, 9778 (2013).

[22] P. Honvault and Y. Scribano, J. Phys. Chem. A 117, 13205 (2013).

[23] E. J. Rackham, F. Huarte-Larranaga, and D. E. Manolopoulos, Chem. Phys. Lett. 343, 356 (2001).

[24] F. J. Aoiz, T. González-Lezana, and V. Sáez-Rábanos, J. Chem. Phys. 127, 174109 (2007).

[25] T. González-Lenzana, P. Honvault, P. G. Jambrina, F. J. Aoiz, and J.-M. Launay, J. Chem. Phys. 131, 044315 (2009).

[26] T. González-Lezana, P. Honvault, and Y. Scribano, J. Chem. Phys. 139, 054301 (2013).

[27] T. González-Lezana, Y. Scribano, and P. Honvault, J. Phys. Chem. A 118, 6416 (2014).

Q [28] D. Gerlich, Adv. Chem. Phys. 82, 1 (1992).

[29] D. Gerlich, R. P. Sil, I. Zymak, M. Hejduk, P. Jusko, D. Mulin, and J. Glosík, J. Phys. Chem. A 117, 10068 (2013).

[30] H. R. Sadeghpour, J. L. Bohn, M. J. Cavagnero, B. D. Esry, I. I. Fabrikant, J. H. Macek, and A. R. P. Rau, J. Phys. B 33 (2000).

[31] M. Przybytek and B. Jeziorski, J. Chem. Phys. 123, 134315 (2005).
[32] W. Vassen, C. Cohen-Tannoudji, M. Leduc, D. Boiron, C. I. Westbrook, A. Truscott, K. Baldwin, G. Birkl, P. Cancio, and M. Trippenbach, Rev. Mod. Phys. 84, 175 (2012).

[33] S. Knoop, P. S. Zuchowski, D. Kedziera, A. Mentel, M. Puchalski, H. P. Mishra, A. S. Flores, and W. Vassen, Phys. Rev. A 90, 022709 (2014).

[34] G. F. Gribakin and V. V. Flambaum, Phys. Rev. A 48, 546 (1993).

[35] L. Velilla, B. Lepetit, A. Aguado, J. A. Beswick, and M. Paniagua, J. Chem. Phys. 129, 084307 (2008).

[36] P. Honvault and J.-M. L. Dynamics, Theory of Chemical Reaction Dynamics NATO Science Series Vol. 145 (Kluwer, New York, 2004).

[37] P. Soldán, M. T. Cvitaš, J. M. Hutson, P. Honvault, and J.-M. Launay, Phys. Rev. Lett. 89, 153201 (2002).

[38] M. Lara, F. Dayou, and J.-M. Launay, Phys. Chem. Chem. Phys. 13, 8359 (2011).

[39] M. Mayle, G. Quéméner, B. P. Ruzic, and J. L. Bohn, Phys. Rev. A 87, 012709 (2013).

[40] M. L. González-Martínez, O. Dulieu, P. Larrégaray, and L. Bonnet, Phys. Rev. A 90, 052716 (2014).

[41] J. Weiner, V. S. Bagnato, S. Zilio, and P. S. Julienne, Rev. Mod. Phys. 71, 1 (1999).

[42] Z. Idziaszek, A. Simoni, T. Calarco, and P. S. Julienne, New J. Phys. 13, 083005 (2011).

[43] B. Gao, Phys. Rev. A 83, 062712 (2011).

[44] J. M. Hutson, New J. Phys. 9, 152 (2007). 